\title{
Ion Temperature Fluctuations in ELMy H-mode of the X3 EC-heated Plasmas on TCV
}

\author{
A.N.Karpushov, B.P.Duval, Ch.Schlatter \\ Ecole Polytechnique Fédérale de Lausanne (EPFL), Centre de Recherches en Physique des Plasmas, \\ Association Euratom-Confédération Suisse, CH-1015 Lausanne, Switzerland
}

\begin{abstract}
This paper focuses on interpreting variations in the NPA measured energy distribution of neutral fluxes from the TCV high density H-mode plasma discharges with strong third harmonic electron cyclotron heating $\left(\mathrm{P}_{\mathrm{X} 3}>\mathrm{P}_{\Omega}\right)$. Two quasi-stationary regimes: ELMy H-mode and ELM-free H-mode, routinely and reproducibly obtained in TCV, with a plasma density $5-10 \times 10^{19} \mathrm{~m}^{-3}$, electron temperature $2-3 \mathrm{keV}$ and ion temperature of $0.7-1.0 \mathrm{keV}$. The ELMy X3-heated $\mathrm{H}$-mode plasma on TCV is significantly perturbed by ELMs, sawteeth activity and modes. In X3-heated plasmas ELMs are characterised by increased amplitudes and lower frequencies than are typical in ohmic H-modes with strong sawteeth synchronised with ELM cycle. The energy losses per ELM can exceed the $15 \%$ of the total stored energy and the plasma density and electron temperature profiles were resolved during the ELM cycle. NPA measurements in the presence of ELMs and sawteeth cannot be explained with the classical theory of two-body Coulomb electron-ion collisions alone. Additional effects (such as a modification of the ion temperature radial profile and/or ion redistribution in the coordinate and velocity space due to plasma perturbations) must be considered.
\end{abstract}

Keywords: Tokamak plasma, H-mode, ELMs, Neural particle analyser, Ion temperature.

PACS: 52.55.Fa, 52.70.Nc, 52.25.Xz, 52.35.Py, 52.50.Sw

\section{INTRODUCTION}

The Tokamak à Configuration Variable (TCV) [1,2] is a medium-sized tokamak $\quad\left(\mathrm{R}=0.88 \mathrm{~m}, \quad \mathrm{a}=0.25 \mathrm{~m}, \quad \mathrm{I}_{\mathrm{p}}<1 \mathrm{MA}, \quad \mathrm{B}_{\mathrm{T}}<1.45 \mathrm{~T}\right.$, plasma elongation 1-2.8) able to produce a plasma with the central electron density $0.5-15 \times 10^{19} \mathrm{~m}^{-3}$, an electron temperature over $10 \mathrm{keV}$ (with X2 $\mathrm{ECH}$ heating) and bulk central ion temperatures of $0.25-1.0 \mathrm{keV}$. TCV is equipped with $3 \mathrm{MW}$ of electron cyclotron heating $(\mathrm{ECH})$ power at the second harmonic (X2: X-mode; $87.4 \mathrm{GHz}, 6$ gyrotrons, low field side launch) and $1.5 \mathrm{MW}$ of a third harmonic ECH system (X3: Xmode, $118 \mathrm{GHz}, 3$ gyrotrons, top launch).

The operational domain of the H-mode plasma with and without edge localised modes (ELMs) has already been reported in [3]. The low-density $\left(\mathrm{n}_{\mathrm{e}} \sim 4.2 \times 10^{19} \mathrm{~m}^{-3}\right)$ cut-off of $\mathrm{X} 2$ waves, prohibits exploring $\mathrm{H}$-modes with $\mathrm{X} 2$ heating, where the working density is often too high. The higher cut-off density for $\mathrm{X} 3$ waves, $\mathrm{n}_{\mathrm{e}} \sim 11 \times 10^{19} \mathrm{~m}^{-3}$, permits the study of auxiliary heated H-modes on TCV. In the contrast with limited electron and ion temperatures coupling in low density $\mathrm{X} 2$ heated TCV plasmas $\left(T_{e} / T_{i}\right.$ in the range of 5-30) X3 ECH heated discharges show increases in both (electron and ion temperatures) from $\sim 1.0 / 0.5 \mathrm{keV}$ to 2-3/0.7-1.0 keV, respectively $[4,5]$.

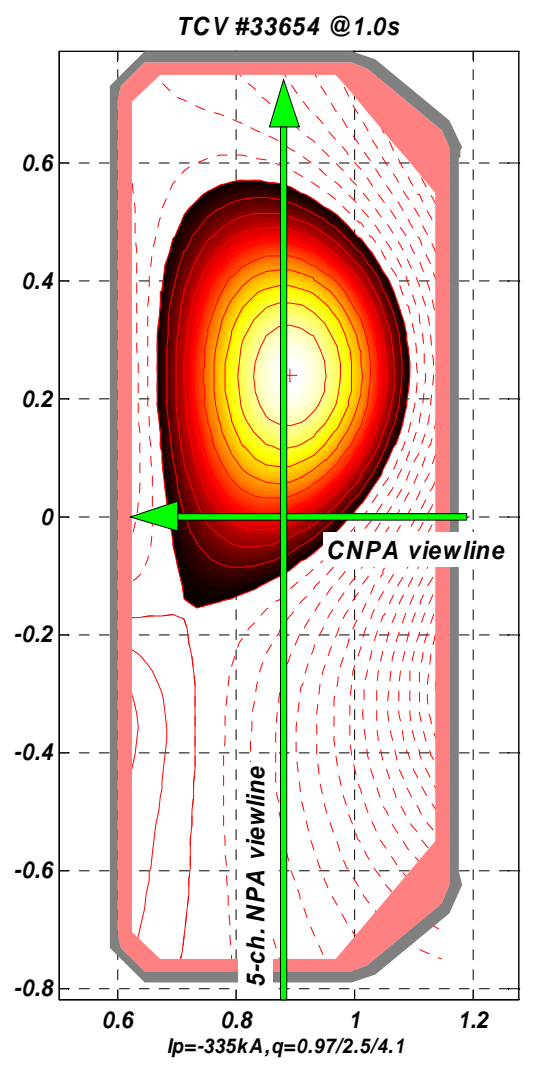

FIGURE 1. Poloidal cross section of the TCV Plasma configuration. 
The EC power deposition and current drive profiles are calculated using the TORAY ray-tracing code with magnetic equilibrium obtained from the LIUQE reconstruction code using measured Thomson scattering (TS) electron temperature and density profiles [6]. The total plasma energy content on TCV was calculated from a diamagnetic loop diagnostic.

Two Neutral Particle Analysers (NPAs) are used on the TCV to obtain energy spectra of energetic neutrals escaping plasma resulting from the charge exchange of plasma ions with neutrals in the plasma. A "Five-Channel Energy Analyser of Atomic Particles" (5-ch.NPA) [7], operated on TCV in double electrical analysis mode, detects particles without atomic mass discrimination in the energy range $0.6-6.5 \mathrm{keV}$, with a time resolution up to $50 \mu \mathrm{s}$. A 28-channel "Compact Neutral Particle Analyser" (CNPA) [7] with mass and energy separation in $\mathrm{E} \| \mathrm{B}$ field simultaneously detects two mass species (11 channels for hydrogen and 17 for deuterium) in the $0.5-50 \mathrm{keV}$ energy range with a time resolution in the $0.5-4.0 \mathrm{~ms}$ range. The DOUBLETCV numerical code is used to model the energy spectra of neutrals leaving the plasma and entering the NPAs [8]. The main carbon impurity $\mathrm{C}^{\mathrm{VI}}$ ion temperature radial profiles were available from the CXRS [9] diagnostic. To improve the statistical accuracy, a coherent averaging technique [10] over several ELM events is been used in the analysis.

\section{X3-HEATED ELMY H-MODE PLASMA}

An Ohmic ELMy H-mode plasma in single null down diverted magnetic configuration (Fig. 1), with $\sim 220 \mathrm{~kW}$ of $\mathrm{OH}$ power and electron density of $5-7 \times 10^{19} \mathrm{~m}^{-3}$, was used as target for X3 heating. 700-800 kW (two gyrotrons) of X3 EC power was used to sustain quasi-stationary plasma conditions, where an increase of the X3 power typically provokes a transition to an ELM-free regime [5]. The plasma current was $300-350 \mathrm{kA}$ and the electron temperature $1.9-2.1 \mathrm{keV}$ before an ELM (and sawteeth crash) falling to $1.2-1.6 \mathrm{keV}$ afterwards. Figure 2 shows some of the main plasma

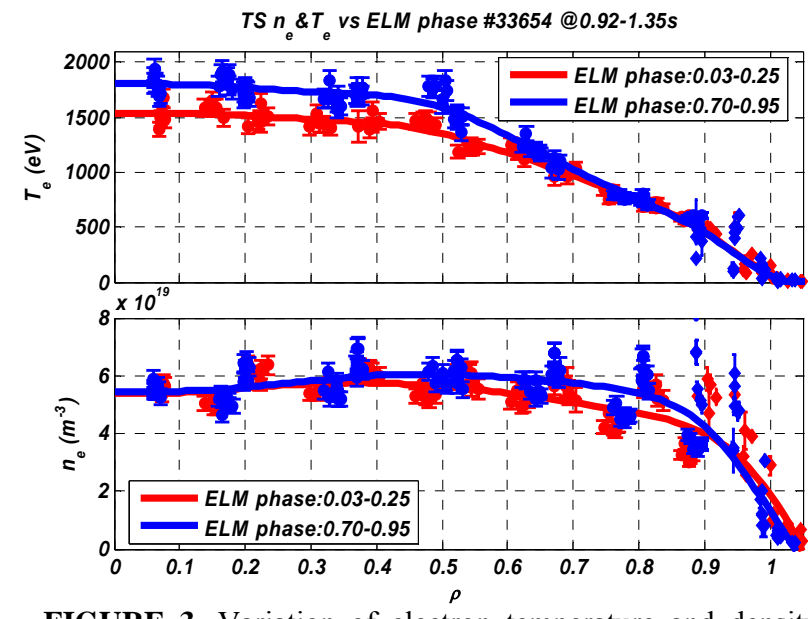

FIGURE 3. Variation of electron temperature and density profiles during the ELM phase

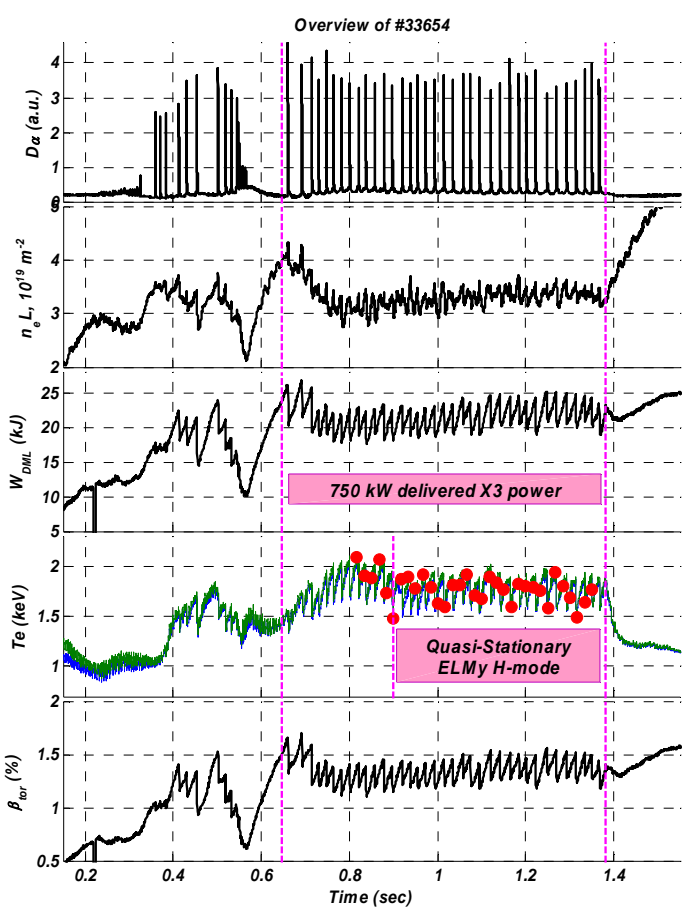

FIGURE 2. Overview of TCV shot 33654 with quasistationary ELMy H-mode with X3 ECH. From top to bottom: the $\mathrm{D}_{\alpha}$ light, line integrated density (vertical view line), the stored energy (magnetic - DML), electron temperature (Soft X-ray foil absorption flux lines, Thomson scattering - dots) and $\beta_{\text {tor }}$.

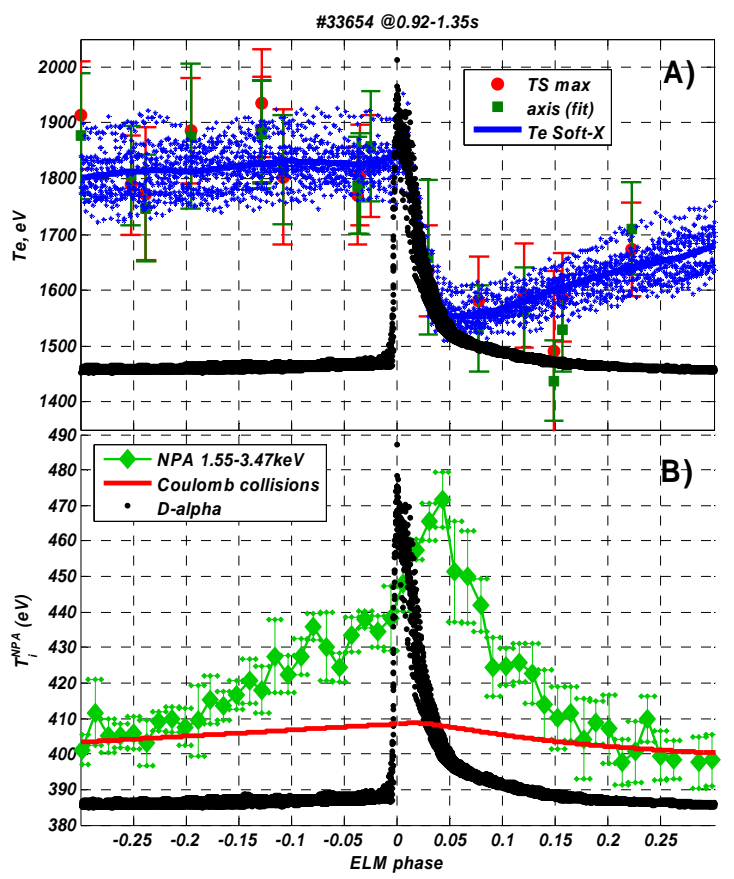

FIGURE 4. Electron temperature (A) and $\mathrm{T}_{\mathrm{i}}^{\mathrm{NPA}}$ (B) variation during ELM-phase: "0" on x-axis - ELM, maximum of $\mathrm{D}_{\alpha}$ emission; -1 - previous ELM; 1 - next $\mathrm{ELM}, \sim 20.5 \mathrm{~ms}$. 
parameters in a TCV discharge with a quasi-stationary ELMy H-mode phase (0.92-1.35 sec). Strong sawteeth activity together with ELMs with a period of $20.5 \pm 2.6 \mathrm{~ms}$ result in a loss of $\sim 15-20 \%$ of the total plasma energy per ELM (values in the range 4-5\% being typical for ohmic H-mode ELMs). According to TS [6] measurement (Fig.3) ELMs leads to a small $(<15 \%)$ decrease of electron density at intermediates plasma radii $(0.3<\rho<0.9)$ and to a fast $(\sim 2 \mathrm{~ms})$ decrease of core electron temperature from $1.8-2.0$ to $\sim 1.5 \mathrm{keV}$ followed by a relatively slow $(\sim 10 \mathrm{~ms})$ recovery time (Fig.4-A). The edge electron temperature profiles do not change significantly (Fig.3).

\section{NPA MEASUREMENT}

The technique described in [7], and references within, has been used for interpretation of the NPA data. In this paper we use a "CX spectra" defined as

$$
F_{d c}(E)=J(E) /\left(\sigma_{c x}(E) \times E\right),
$$

where $\sigma_{c x}$ is the cross-section for charge exchange. The energy spectra of the passive atomic flux $J(E)$ emitted from the plasma into the NPA is the sum of fluxes in the plasma column along the view line of the analyser :

$$
J(E)=\Omega \cdot S \cdot \int_{-a}^{a} n_{a} \cdot n_{i} \cdot f_{i}(E) \cdot\left\langle\sigma_{c x}\left(v_{i a}\right) \cdot v_{i a}\right\rangle \cdot \gamma \cdot d z
$$

$J(E)$ is proportional to the number of pulses detected by a given NPA channel (count-rate) $N(E)$ : $J(E)=N(E) /\left(\Delta t \cdot \Delta E \cdot \alpha_{\text {det }}(E)\right)$. The temporal evolution of NPAs count-rates at several energies in quasistationary ELMy phase are shown in Fig.5. Fast ELM-driven $(<1 \mathrm{~ms})$ flux-spikes are clearly distinguishable from the 5-ch. NPA followed by decrease in the count-rates with a few ms decay time to below pre-ELM values. The increase of neutral density caused by the ELM related transient increase in the plasma-wall interaction is sufficient to explain these temporal flux-spikes.

For low density plasmas with a Maxwellian ion energy distribution, the ion temperature should be proportional to the logarithm of the $F_{d c}$ slope; in most situations, including ELMy H-mode on the TCV, the plasma does not exhibit a single ion temperature and since attenuation may not be neglected the ion temperature, inferred from the slope of $\left(F_{d c}\right)$, depends on the neutrals' energy:

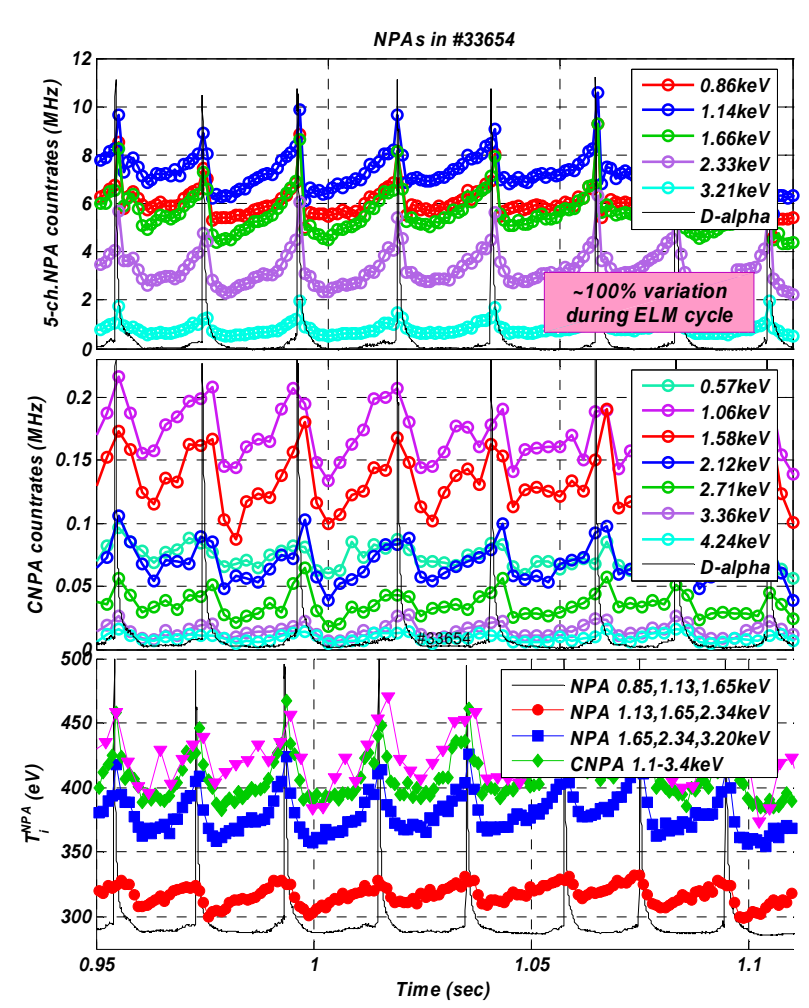

FIGURE 5. Variation of NPAs count-rates and $\mathrm{T}_{\mathrm{i}}^{\mathrm{NPA}}$ during a quasi-stationary ELMy phase.

$$
1 / T_{i}^{N P A}(E)=d \ln \left(F_{d c}\right) / d E
$$

$T_{i}^{N P A}$ from the NPA CX spectrum, with TCV plasma conditions typical for ELMy H-mode, is 1.5-2 times lower than core ion temperature (Fig.6). There is a significantly larger variation of the neutral fluxes at high energy $(>2.5 \mathrm{keV})$ than for lower energies (characterised by $T_{i}^{N P A}$ ) during an ELM cycle (Fig.5, 4-B).

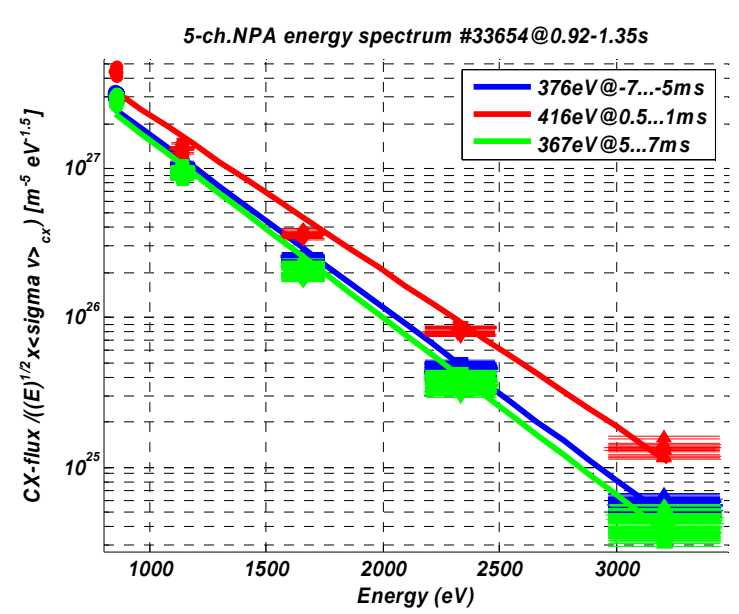

FIGURE 6. Measured energy distribution function ("NPA CX-spectra") before $(-7 \ldots-5 \mathrm{~ms})$, during $(0.5 \ldots 1 \mathrm{~ms})$ and $\operatorname{after}(5 \ldots 7 \mathrm{~ms})$ ELM. 


\section{DISCUSSION}

The correlation of the neutral flux $(J(E)$, eq.2) with ELMs could be caused by a several plasma effects: (a) increase of neutral density $\left(n_{a}\right)$ due to ELM related transient increase of plasma-wall interaction; (b) plasma density $\left(n_{i}\right)$ variation; (c) variation of ion temperature $\left(T_{i}\right)$ due to variation of electron temperature and power exchange between electrons and ions (Coulomb collisions); (d) modification of ion energy distribution function $\left(f_{i}\left(v^{3}, \rho\right)\right)$ due to ion redistribution in coordinate and/or velocity space. DOUBLE-TCV simulations [8] show that for TCV ELMy H-mode discharges neutral fluxes with low $(<1 \mathrm{keV})$ energies originate from the plasma edge (emissivity function have a maximum at $\rho \subseteq[0.85 \ldots 1.0])$, whereas high energy fluxes originate from the plasma core (intermediate plasma radii, $\rho \subseteq[0.75 \ldots 0.85]$ for $3 \mathrm{keV})$. The transient increase of neutralisation probability should result in a stronger and faster increase of the neutral fluxes at lower energies (whereas experimental observations are opposite) and can thus not explain the increase of $T_{i}^{N P A}$ during ELM cycle and the dependence of temporal variation of neutral fluxes with different energies (Fig.5,7). A plasma density variation of $10-15 \%$ is not sufficient to explain the observed increase in the neutral flux from decreased attenuation of the escaping plasma neutrals (increase of probability, $\gamma$ ). An analysis of the temporal behaviour of the Coulomb collisional electron-ion power balance indicates only a 2-3\% change of ion temperature mainly due the electron temperature variation (Fig.4). The variation of the $T_{i}^{N P A}$ during ELM cycle (Fig.4,5) is much stronger corresponding to a $10-15 \%$ increase in $T_{i}$.

An ELM (or/and sawteeth) induced redistribution of ion remains the only considered candidate to explain NPA observations in the TCV ELMy H-mode plasma. Sawteeth crashes and ELMs are accompanied by a variation of the axial safety factor $\left(q_{0}\right)$ around 1 and plasma vertical and radial displacements of a few mm. Modes excitation could then result in strong ion mixing, especially of trapped ions. A set of experiments with NPA observations at different toroidal angles of view line, various q-profiles, etc. is planned for the upcoming TCV experimental campaign.

\section{ACKNOWLEDGMENTS}

The authors are grateful to Y.Martin and L.Porte for coordination of the TCV experimental program on "X3 heated H-mode plasma", and R.Behn for support in analysis of Thomson scattering data.

This work was partly supported by the Swiss National Science Foundation.

\section{REFERENCES}

1. F. Hofmann et al., Plasma Phys. Controlled Fusion 36, B277-B287 (1994).

2. T. Goodman et al., Nucl. Fusion 43, 1619-1631 (2003).

3. A.W. Degeling, et al., Plasma Phys. Control. Fusion 43, 1671-1698 (2001)

4. Y.R. Martin, L. Porte and S. Alberti, Plasma Phys. Control. Fusion 48, A163-A169 (2006)

5. L. Porte and the TCV Team, Proc. 17th Topical Conference on Radio Frequency Power in Plasmas, Clearwater (Florida) 2007, AIP Conference Proceedings, Volume 933, pp.385-392 (2007)

6. R.Behn et al,. Plasma Phys. Control. Fusion 49, 1289-1308 (2007)

7. A.N. Karpushov, et al., Rev. Sci. Instrum 77, 033504 (2006)

8. Ch. Schlatter, B.P. Duval and A.N. Karpushov, Plasma Phys. Control. Fusion 48, 1765-1785(2006)

9. P. Bosshard, et al.,, Proc. 29th EPS Conference on Controlled Fusion and Plasma Physics, Montreux, Switzerland, June 2002, ECA Vol. 26B, P-4.120 (2002)

10. H.F. Tammen, et.al., Rev. Sci. Instrum 66(1), 327-329 (1995) 